\title{
Involvement of the phosphoinositide 3-kinase/Akt pathway in apoptosis induced by capsaicin in the human pancreatic cancer cell line PANC-1
}

\author{
JIAN-HONG ZHANG $^{1 *}$, FU-JI LAI $^{1 *}$, HUI CHEN $^{1 *}$, JIANG LUO $^{1}$, RI-YUAN ZHANG ${ }^{1}$, \\ HE-QI BU ${ }^{1}$, ZHAO-HONG WANG ${ }^{1}$, HONG-HAI LIN ${ }^{1}$ and SHENG-ZHANG LIN ${ }^{1,2}$ \\ ${ }^{1}$ Department of Hepatobiliary-Pancreatic Surgery, The Second Affiliated Hospital of Wenzhou Medical College, \\ Wenzhou, Zhejiang 325027; ${ }^{2}$ Department of Hepatobiliary-Pancreatic Surgery, First Affiliated Hospital, \\ Zhejiang University School of Medicine, Hangzhou 310003, P.R. China
}

Received July 23, 2012; Accepted October 11, 2012

DOI: $10.3892 / \mathrm{ol} .2012 .991$

\begin{abstract}
Capsaicin, one of the major pungent ingredients found in red peppers, has been recently demonstrated to induce apoptosis in various malignant cell lines through an unclear mechanism. In this study, the effect of capsaicin on proliferation and apoptosis in the human pancreatic cancer cell line PANC-1 and its possible mechanism(s) of action were investigated. The results of a Cell Counting Kit-8 (CCK-8) assay revealed that capsaicin significantly decreased the viability of PANC-1 cells in a dose-dependent manner. Capsaicin induced G0/G1 phase cell cycle arrest and apoptosis in PANC-1 cells as demonstrated by a flow cytometric assessment. Caspase-3 expression at both the protein and mRNA level was promoted following capsaicin treatment. Furthermore, we revealed that phospho-PI3 Kinase p85 (Tyr458) and phospho-Akt (Ser473) in PANC-1 cells were downregulated in response to capsaicin. Moreover, capsaicin gavage significantly inhibited the growth of pancreatic cancer PANC-1 cell xenografts in athymic nude mice. An increased number of TUNEL-positive cells and cleaved caspase-3 were observed in capsaicin-treated mice. In vivo, capsaicin downregulated the expression of phospho-PI3 Kinase p85 (Tyr458) and phospho-Akt (Ser473). In conclusion, we have demonstrated that capsaicin is an inhibitor of growth of PANC-1 cells, and downregulation of the phosphoinositide 3-kinase/Akt pathway may be involved in capsaicin-induced apoptosis in vitro and in vivo.
\end{abstract}

Correspondence to: Dr Sheng-Zhang Lin, Department of Hepatobiliary-Pancreatic Surgery, The Second Affiliated Hospital of Wenzhou Medical College, 109 Xue-yuan Road, Wenzhou, Zhejiang 325027, P.R. China

E-mail:wzf21sz@163.com

${ }^{*}$ Contributed equally

Key words: capsaicin, pancreatic cancer, apoptosis, PI3K/Akt pathway

\section{Introduction}

Pancreatic cancer, one of the most fatal types of solid malignancy, is the fourth leading cause of cancer-related mortality in the USA and other industrialized countries, leading to an estimated 227,000 deaths per year worldwide (1,2). Certain studies have demonstrated that the mortality rates of pancreatic cancer in China have constantly increased over the past decade. Presently, it is the eighth-leading cause of cancerrelated mortality in China (3). Due to difficulties in early diagnosis and its highly aggressive malignant behavior, only $10-20 \%$ of pancreatic cancers can be surgically resected with curative intent at the time of diagnosis and the majority of patients experience local recurrence and metastasis (4). Despite therapeutic advances, the prognosis of patients with pancreatic ductal adenocarcinoma is extremely poor; the median survival time is 6 months and less than $5 \%$ survive 5 years following the initial diagnosis $(1,5)$. Gemcitabine is the current standard therapy for advanced pancreatic cancer. However, gemcitabine treatment results in an objective tumor response rate of less than $20 \%$, and only a marginal survival advantage is associated with multiple adverse events and drug resistance $(6,7)$. It is therefore of particular interest to impose new therapeutic strategies and to improve the prognosis of this potentially fatal disease.

The phosphoinositide 3-kinase (PI3K) pathway is a key signal transduction system that links oncogenes and multiple receptor classes to a number of essential cellular functions, and is possibly the most commonly activated signaling pathway in human cancer $(8,9)$. The activation of the PI3K pathway is relatively well understood; it is known to be a multi-step process involving the PI3K-dependent phosphorylation of phospholipids localized at the plasma membrane, and the subsequent membrane localization of phosphoinositide-dependent kinase 1 (PDK1) and Ser/Thr kinase Akt (also known as protein kinase $\mathrm{B}, \mathrm{PKB}$ ) via their pleckstrin homology $(\mathrm{PH})$ domains $(9,10)$. The activation of PI3K ultimately leads to Akt phosphorylation, and activated Akt controls fundamental cellular processes including cell survival by phosphorylating and inactivating several downstream pro-apoptotic target 
molecules (9,11). Another study has demonstrated that the $\mathrm{PI} 3 \mathrm{~K} / \mathrm{Akt}$ pathway is constitutively activated in a majority of human pancreatic cancer cell lines (12). It has been revealed that inhibition of the PI3K/Akt pathway results in inhibition of tumor growth and apoptosis, and the PI3K/Akt pathway is considered as a viable and effective target for pancreatic cancer therapy $(9,12,13)$.

Capsaicin is one of the major pungent ingredients found in red peppers, which are among the most commonly and frequently used spices in the world $(14,15)$. Due to its analgesic activity, topical application of capsaicin has been used in clinical practice for the treatment of neuropathic pain $(16,17)$. Capsaicin has been revealed to inhibit growth and induce apoptosis in various malignant cell lines (18-21). Moreover, capsaicin treatment has significantly suppressed the growth of tumors in athymic nude mice transplanted with cancer cells $(22,23)$. Capsaicin was also revealed to inhibit the PI3K/Akt pathway in B16-F10 melanoma cells (24). However, data regarding the properties of capsaicin in pancreatic cancer cells remain limited and the mechanisms of apoptosis have not yet been fully elucidated. Based on these studies, we hypothesized that capsaicin may exhibit an antitumor effect and induce apoptosis in pancreatic cancer cells, via downregulation of the PI3K/Akt pathway.

In the present study, we show that capsaicin significantly inhibits the growth of PANC-1 cells and triggers apoptosis in a dose-dependent manner. Treatment of PANC-1 cells with capsaicin resulted in downregulation of phospho-PI3 Kinase p85 (Tyr458) and phospho-Akt (Ser473). Furthermore, capsaicin also inhibited the growth of pancreatic cancer PANC-1 xenograft tumors induced in athymic nude mice. These results suggest that capsaicin may be an effective and promising antitumor agent against pancreatic cancer.

\section{Materials and methods}

Reagents and antibodies. Capsaicin, dimethyl sulfoxide (DMSO), and propidium iodide (PI) were obtained from Sigma-Aldrich (St. Louis, MO, USA). RNase was obtained from Fermentas (St. Leon-Rot, Germany). Dulbecco's modified Eagle's medium (DMEM), fetal bovine serum (FBS), penicillin-streptomycin and trypsin-EDTA were obtained from Gibco BRL (Invitrogen, Grand Island, NY, USA). Rabbit cleaved caspase-3 antibody, phospho-PI3 Kinase p85 (Tyr458) antibody, phospho-Akt (Ser473) antibody and $\beta$-tubulin antibody were purchased from Cell Signaling Technology, Inc. (Beverly, MA, USA). Horseradish peroxidase (HRP)conjugated goat anti-rabbit secondary antibody was purchased from Beyotime Biotechnology (Haimen, China).

Cell culture. The human pancreatic cancer cell line PANC-1 was purchased from Shanghai Cell Bank (Shanghai, China). The cell line was maintained in continuous exponential growth in DMEM supplemented with $10 \% \mathrm{FBS}, 100 \mathrm{U} / \mathrm{ml}$ penicillin and $100 \mu \mathrm{g} / \mathrm{ml}$ streptomycin, at $37^{\circ} \mathrm{C}$ under a humidified $5 \% \mathrm{CO}_{2}$ atmosphere.

Cell viability. PANC-1 cells were seeded at a density of $5 \times 10^{3} /$ well in 96 -well plates. Following incubation overnight, the medium was removed and replaced with fresh medium containing different concentrations of capsaicin $(50,100,150$, 200,250 or $300 \mu \mathrm{M}$ ) or DMSO (control) for $24 \mathrm{~h}$. On completion of incubation, cell viability was determined using Cell Counting Kit-8 (CCK-8; Dojindo Molecular Technologies, Kumamoto, Japan) according to the manufacturer's instructions. CCK- 8 reagent $(10 \mu \mathrm{l})$ was added to $100 \mu \mathrm{l}$ media in each well and incubation was continued for a further $3 \mathrm{~h}$. The absorbance (A) of each well was read at $450 \mathrm{~nm}$ using an enzyme-linked immunosorbant assay (ELISA) reader (Bio-Tek ELx808, Winooski, VT, USA). Percentage suvival rate was calculated using the following equation: Survival rate $(\%)=\left(\mathrm{A}_{\text {sample }}-\mathrm{A}_{\text {blank }}\right) /\left(\mathrm{A}_{\text {control }}-\mathrm{A}_{\text {blank }}\right)$.

Cell cycle analysis. PANC-1 cells were seeded at a density of approximately $5 \times 10^{5}$ cells/well into 6 -well plates, cultured overnight and then 150,200 or $250 \mu \mathrm{M}$ capsaicin or DMSO (control) was added. Following $24 \mathrm{~h}$ of incubation, cells were harvested, washed with PBS and then fixed with $70 \%$ ethanol overnight at $4^{\circ} \mathrm{C}$. Cells were stained with $20 \mu \mathrm{g} / \mathrm{ml}$ RNase and $20 \mu \mathrm{g} / \mathrm{ml} \mathrm{PI}$ for $30 \mathrm{~min}$ at $37^{\circ} \mathrm{C}$ in the dark, and then analyzed by flow cytometry (Becton-Dickinson, San Jose, CA, USA).

Apoptosis assay. The measurement of phosphatidylserine redistribution in a plasma membrane was conducted according to the manufacturer's instructions for the Annexin V-FITC/ PI Apoptosis Detection kit (BioVision, Mountain View, CA, USA). Following 150,200 or $250 \mu \mathrm{M}$ capsaicin or DMSO (control) treatment, harvested cells were suspended in $500 \mu \mathrm{l}$ Annexin $\mathrm{V}$ binding buffer. Then, $5 \mu \mathrm{l}$ Annexin V-FITC and $10 \mu 1$ PI were added and incubated with the cells for $5 \mathrm{~min}$ in the dark. The stained cells were analyzed directly by flow cytometry using the Cell Quest program (Becton-Dickinson).

Western blot analysis. PANC-1 cells were treated with 150, 200 or $250 \mu \mathrm{M}$ capsaicin or DMSO (control). Following incubation, the cells were lysed in Cell Lysis buffer $(20 \mathrm{mM}$ Tris-HCl pH 7.5, $150 \mathrm{mM} \mathrm{NaCl}, 1 \mathrm{mM} \mathrm{Na}{ }_{2}$ EDTA, $1 \mathrm{mM}$ EGTA, 1\% Triton, $2.5 \mathrm{mM}$ sodium pyrophosphate, $1 \mathrm{mM}$ beta-glycerophosphate, $1 \mathrm{mM} \mathrm{Na} \mathrm{VO}_{4}, 1 \mu \mathrm{g} / \mathrm{ml}$ leupeptin and $1 \mathrm{mM}$ PMSF; Cell Signaling Technology, Beverly, MA, USA) for $5 \mathrm{~min}$ on ice and then subjected to sonication for 20 sec. Protein concentrations were measured using the BCA Protein Assay kit (Pierce Biotechnology, Inc., Rockford, IL, USA). Equal amounts of protein were separated on 8 or $12 \%$ SDS-PAGE, and transferred onto a polyvinylidene difluoride membrane. Next, the membrane was blocked with $5 \%$ BSA and then incubated overnight with cleaved caspase-3 antibody, phospho-PI3 Kinase p85 (Tyr458) antibody or phospho-Akt (Ser473) antibody. Following extensive washing, the membrane was incubated with appropriate secondary antibodies conjugated with horseradish peroxidase for $1 \mathrm{~h}$ at room temperature. Following washing, immunoblots were developed using the Enhanced Chemiluminescence kit (Pierce).

Real-time polymerase cahin reaction (PCR). PANC-1 cells were treated with $200 \mu \mathrm{M}$ capsaicin or DMSO (control) for $24 \mathrm{~h}$ and then total RNA was isolated from treated cells using TRIzol reagent (Invitrogen). Total RNA ( $1 \mu \mathrm{g})$ was reverse transcribed in $20 \mu \mathrm{l}$ volume, using RevertAid ${ }^{\mathrm{TM}}$ First Strand cDNA Synthesis kit (Fermentas). Reverse transcriptase 
reaction mixture $(1 \mu \mathrm{l})$ was then real-time PCR-amplified in Mastercycler ${ }^{\circledR}$ ep Realplex (Eppendorf, Germany). The initial denaturation step was $95^{\circ} \mathrm{C}$ for $60 \mathrm{sec}$, followed by 40 cycles of amplification at $95^{\circ} \mathrm{C}$ for $15 \mathrm{sec}, 60^{\circ} \mathrm{C}$ for $15 \mathrm{sec}$ and $72^{\circ} \mathrm{C}$ for $45 \mathrm{sec}$. The primers used were: Caspase- 3 forward 5'-CAGTGGAGGCCGACTTCTTG-3' and reverse 5'-TGGCACAAAGCGACTGGAT-3'; RPLP0 forward 5'-GAGACAAAGTGGGAGCCAGCGA-3' and reverse 5'-ACCCTCCAGGAAGCGAGAATGC-3'. All samples were performed in triplicate and the relative quantity of the target gene was normalized with the housekeeping gene RPLP0.

In vivo studies. BALB/c (nu/nu) four-week-old male mice were purchased from Shanghai Laboratory Animals Center (Shanghai, China) and maintained in specific pathogen-free conditions. All animal studies were approved by the Animal Research and Ethical Committee of Wenzhou Medical College (Zhejiang, China). Pancreatic cancer xenograft tumor model was performed as described in our previous studies $(25,26)$. PANC-1 cells $\left(5 \times 10^{6}\right)$ in $200 \mu$ l complete culture medium were injected subcutaneously into the right flank of each mouse. Four weeks after cell inoculation, eight randomized animals for each experimental group received capsaicin $(5 \mathrm{mg} / \mathrm{kg}$ body weight in $100 \mu \mathrm{l}$ of PBS containing $0.3 \%$ ethanol) or vehicle (100 $\mu \mathrm{l}$ of PBS containing $0.3 \%$ ethanol) by gavage three days a week (Monday, Wednesday and Friday) for four weeks. One week after the last treatment, the mice were sacrificed. The tumors were weighed with an electronic balance and tumor volumes were calculated with a vernier caliper using the following formula: $(4 \pi / 3) \times(\text { width } / 2)^{2} \times($ length/2). Half of the tumor tissue in each group was formalin-fixed and paraffin-embedded for TUNEL assay. Remaining tumor tissue was stored in liquid nitrogen for western blot analysis. Western blot analysis in tumor tissue was performed as previously described in vitro.

In situ detection of apoptotic cells in tumor tissues. Apoptotic cells in the tumor tissues were detected by TUNEL assay, according to the manufacturer's instructions for the In Situ Cell Death Detection kit (Roche, Mannheim, Germany). Sections were deparaffinized in xylene and then treated with a graded series of alcohol $(100,95,90,80$ and $70 \%$ ethanol in doubledistilled water) and rehydrated in PBS ( $\mathrm{pH}$ 7.5). Tissues were then treated with proteinase $\mathrm{K}$ solution for permeabilization and treated with TUNEL reaction mixture, then incubated at $37^{\circ} \mathrm{C}$ for $1 \mathrm{~h}$. Apoptotic cells were photographed under a fluorescence microscope (Nikon, Tokyo, Japan).

Statistical analysis. Data are represented as mean \pm standard deviation for the absolute values or percentage of controls. SPSS13.0 software (SPSS, Inc., Chicago, IL, USA) was used for statistical analysis. Differences between the capsaicintreated and DMSO-treated (control) groups were analyzed by an unpaired Student's t-test or ANOVA. $\mathrm{P}<0.05$ was considered to indicate a statistically significant difference.

\section{Results}

Effect of capsaicin on cell proliferation. To investigate the effect of capsaicin on cell growth, PANC-1 cells were treated

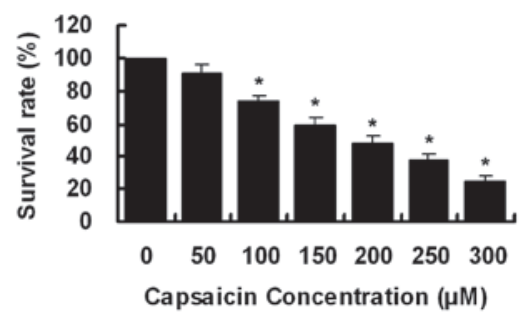

Figure 1. Evaluation of cell viability by Cell Counting Kit-8 (CCK-8) assay as described in Materials and methods. PANC-1 cells were treated with different concentrations of capsaicin for $24 \mathrm{~h}$. On termination of incubation, viable cells were evaluated relative to dimethyl sulfoxide (DMSO)-treated cells (control) and interpreted as percentage of viable cells. Data obtained from three separate experiments are expressed as mean \pm standard deviation and ${ }^{*} \mathrm{P}<0.01$, compared with the control group.

with increasing concentrations of capsaicin $(0-300 \mu \mathrm{M})$ for $24 \mathrm{~h}$. Cell viability was determined by CCK-8 assay. As demonstrated in Fig. 1, cell growth was inhibited by capsaicin treatment in a dose-dependent manner with an $\mathrm{IC}_{50}$ of $\sim 200 \mu \mathrm{M}$.

Capsaicin induces $60 / G 1$ phase arrest in PANC-1 cells. We investigated whether the antiproliferative activity of capsaicin in PANC-1 cells was correlated with cell cycle arrest. As demonstrated in Fig. 2, following capsaicin treatment, cell cycle analysis revealed that capsaicin increased the number of cells in the G0/G1 phase in a dose-dependent manner.

Capsaicin triggers apoptosis in PANC-1 cells. As demonstrated in Fig. 3A, treatment with capsaicin induced a greater level of apoptosis in PANC-1 cells, as revealed by flow cytometric assessment. Apoptotic rates in $250 \mu \mathrm{M}$ capsaicin-treated cells and control cells were $19.95 \pm 0.76 \%$ and $10.21 \pm 0.45 \%$, respectively. As demonstrated in Fig. 3B, a dose-dependent increase in the cleaved caspase- 3 was observed after the exposure of cells to increasing concentrations of capsaicin. Compared with DMSO-treated cells, the level of caspase- 3 mRNA expression was higher (1.44-fold) following $200 \mu \mathrm{M}$ capsaicin treatment (Fig. 3C).

Downregulation of PI3K/Akt pathway by capsaicin in PANC-1 cells. To elucidate the mechanism of antiproliferation and apoptosis of capsaicin in PANC-1 cells, we employed western blot analysis for phospho-PI3 Kinase p85 (Tyr458) and phospho-Akt (Ser473). As demonstrated in Fig. 4, capsaicin significantly downregulated the expression of phospho-PI3 Kinase p85 (Tyr458) and phospho-Akt (Ser473) in a dosedependent manner.

Antitumoral effect of capsaicin in vivo. To investigate the antitumoral effect of capsaicin on pancreatic cancer cells in vivo, we first generated pancreatic cancer xenograft tumors in athymic nude mice. One week after the last treatment, the mice were sacrificed and the tumors were weighed. Tumor volumes were also measured. As demonstrated in Fig. 5A, the weights of tumors in vehicle-treated mice were $\sim 1.43$-fold greater than that of capsaicin-treated mice. Tumor volumes in capsaicin-treated mice and vehicle-treated mice were $617.25 \pm 85.07 \mathrm{~mm}^{3}$ and 

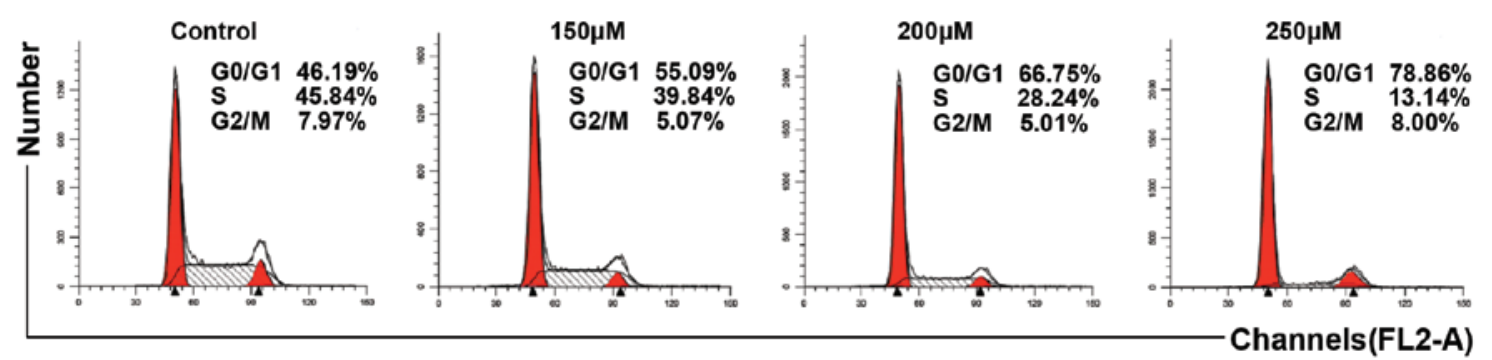

Figure 2. Capsaicin induces G0/G1 phase arrest. Treated cells were stained with propidium iodide (PI) and DNA content was analyzed by flow cytometry. The number of cells in each phase was calculated using ModFit software. The results shown are representative of three independent experiments.
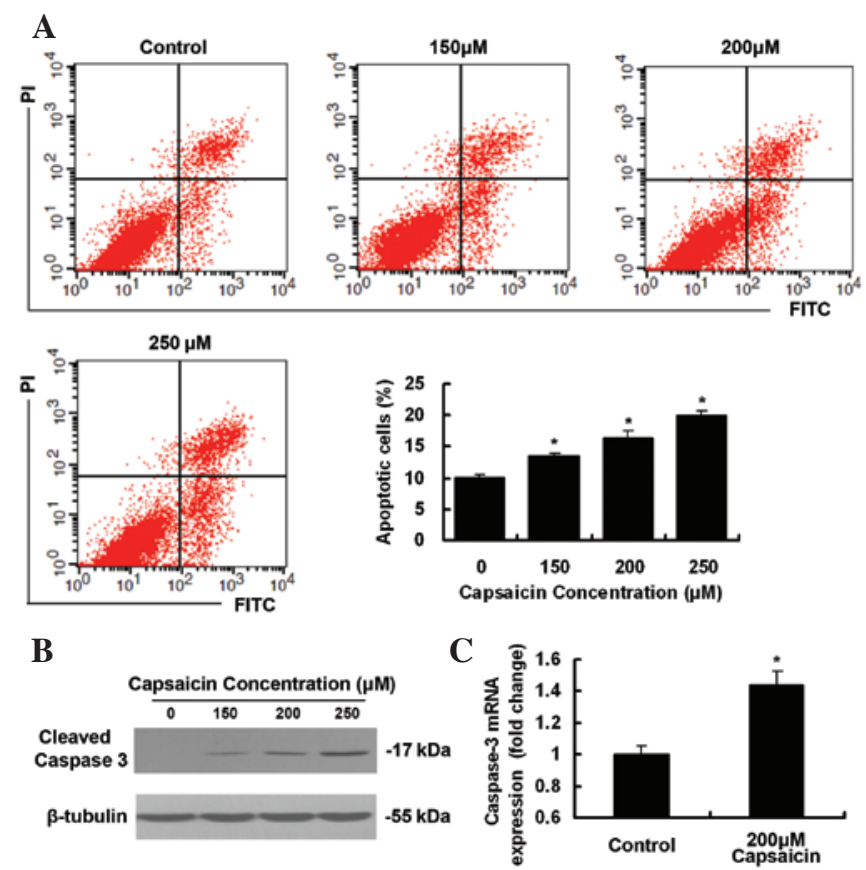

Figure 3. Capsaicin triggers apoptosis in PANC-1 cells. (A) Apoptotic cells were assessed by qualitative flow cytometry using Annexin $\mathrm{V}$ and propidium iodide (PI) staining. Cells in the lower left quadrant (Annexin V-FITC- $/ \mathrm{PI}^{-}$) are viable, those in the lower right quadrant (Annexin $\left.\mathrm{V}-\mathrm{FITC}^{+} / \mathrm{PI}\right)$ are early apoptotic and those in the upper right quadrants (Annexin $\mathrm{V}-\mathrm{FITC}^{+} / \mathrm{PI}^{+}$) are late apoptotic or necrotic. Data obtained from three separate experiments are expressed as mean \pm standard deviation $(\mathrm{SD})$ and ${ }^{*} \mathrm{P}<0.01$, compared with the control group. (B) Cleaved caspase-3 examined by western blot analysis. $\beta$-tubulin was used as a loading control. Results demonstrated are representative of three independent experiments. (C) mRNA expression of caspase- 3 examined by real-time PCR. The relative quantity of the target gene was normalized with the housekeeping gene RPLP0. Data are expressed as mean $\pm \mathrm{SD}$ and ${ }^{*} \mathrm{P}<0.01$, compared with control group.

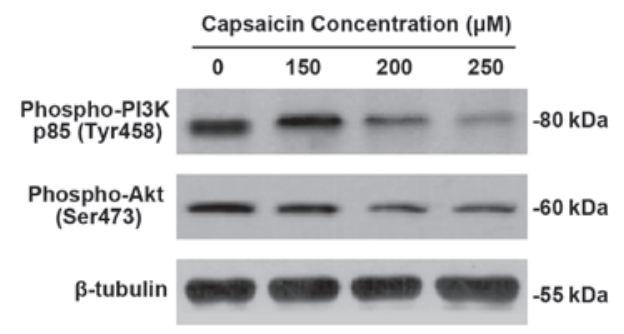

Figure 4. Effect of capsaicin on protein expression of phospho-PI3 Kinase p85 (Tyr458) and phospho-Akt (Ser473) in PANC-1 cells. Western blot analysis was performed as described in Materials and methods. $\beta$-tubulin was used as a loading control. Results demonstrated are representative of three independent experiments.
A

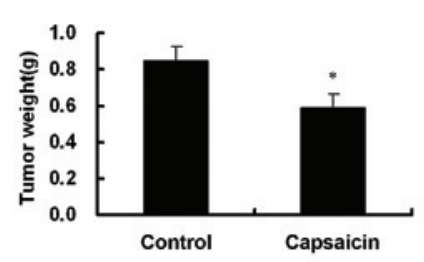

B

C

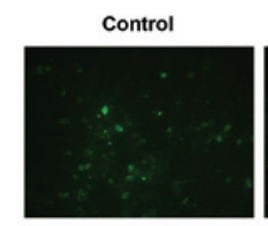

Capsaicin

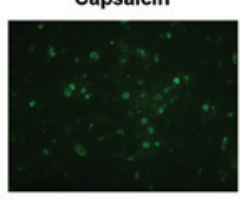

D

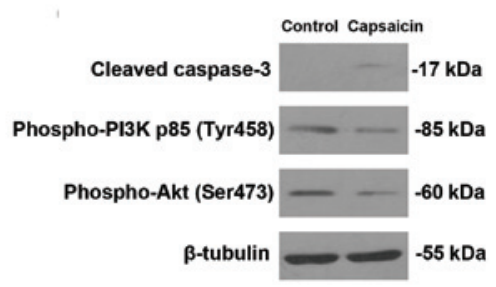

Figure 5. Effect of capsaicin on a pancreatic cancer xenograft in nude mice. (A) Tumor weights. Data are expressed as mean \pm standard deviation (SD) and ${ }^{*} \mathrm{P}<0.05$, compared with vehicle-treated mice. (B) Tumor volumes. Data are expressed as mean $\pm \mathrm{SD}$ and ${ }^{*} \mathrm{P}<0.05$, compared with vehicle-treated mice. (C) Apoptotic cells evaluated by TUNEL assay in tumor tissues. (D) Western blot analysis for cleaved caspase-3, phospho-PI3 Kinase p85 (Tyr458) and phospho-Akt (Ser473). $\beta$-tubulin was used as a loading control. The results shown are representative of three independent experiments.

$921.16 \pm 110.29 \mathrm{~mm}^{3}$, respectively (Fig. 5B). As demonstrated in Fig. 5C, increased TUNEL-positive cells were observed in capsaicin-treated mice. Consistent with our studies in vitro, cleaved caspase-3 was observed in capsaicin-treated mice and capsaicin downregulated the expression of phospho-PI3 Kinase p85 (Tyr458) and phospho-Akt (Ser473) (Fig. 5D).

\section{Discussion}

Epidemiologic studies have revealed that several dietary agents modulate diverse biochemical processes involved in carcinogenesis (27). These include inhibition of carcinogen activation, cellular proliferation and tumor metastasis, blockading tumor cell cycle progression and induction of apoptosis (27). In vitro 
and in vivo studies have shown that dietary chemopreventive agents may serve as potent agents for enhancing the therapeutic effects of chemotherapy, radiotherapy or other standard therapeutics for the treatment of human cancers (28). Capsaicin is the principal pungent ingredient present in red peppers, which are among the most frequently consumed spices worldwide $(14,15)$. Capsaicin has been revealed to possess inhibitory effects in various cancer cells (18-20,22,24). However, the precise molecular mechanisms have not been well elucidated in pancreatic cancer cells. In this study, we first used a CCK-8 assay to detect cell viability following capsaicin treatment. The results revealed that capsaicin significantly inhibited PANC-1 cell proliferation in a dose-dependent manner. Furthermore, capsaicin gavage significantly inhibited the growth of pancreatic cancer PANC-1 cell xenografts in athymic nude mice.

Next, we investigated whether the antiproliferative activity of capsaicin in PANC-1 cells was due to cell cycle arrest and apoptosis. In the present study, capsaicin treatment in PANC-1 cells induced G0/G1 phase arrest and apoptosis in a dosedependent manner. The results of the TUNEL assay revealed that increased numbers of apoptotic cells were observed in capsaicin-treated mice. Previously, accumulated evidence indicated that caspases, a family of cysteine proteases, play a pivotal role in the apoptotic process; caspase-3 is an apoptosis executioner and is activated by other activated caspases, including caspase-8 and 9 (29,30). Activated caspase-3 subsequently cleaves certain specific substrates, including poly (ADP-ribosyl) polymerase (PARP) and D4-GDI proteins, which are important for the occurrence of typical biochemical and morphological changes in apoptotic cells $(29,31)$. To further confirm that the antiproliferative activity of capsaicin was due to apoptosis, we examined caspase-3 activation, an event that is commonly used as a hallmark of apoptosis. In this study, caspase-3 was activated after capsaicin treatment in vitro and in vivo. These results suggest that apoptosis may be a potential general mechanism and provide a mechanistic basis for the antiproliferative as well as anti-neoplastic effects of capsaicin in PANC-1 cells.

To further elucidate the mechanism of antiproliferation and apoptosis of capsaicin in PANC-1 cells, we investigated the PI3K/Akt pathway. It has been demonstrated that the PI3K/ Akt signaling pathway components are frequently altered in human cancers and inappropriately activated $(9,11)$. PI3Ks are divided into three classes according to their structural characteristics and substrate specificity. Of these, the most commonly studied are the class I enzymes that are activated directly by cell surface receptors. Class I PI3Ks are further divided into class IA enzymes, which are activated by RTKs, GPCRs and certain oncogenes including the small $G$ protein RAS, and class IB enzymes, which are regulated exclusively by GPCRs. Class IA PI3Ks are heterodimers consisting of a p110 catalytic subunit and a p85 regulatory subunit $(8,9)$. In the present study, the phosphorylation level of PI3K at Tyr458 of the p85 regulatory subunit was significantly reduced in capsaicin-treated cells as compared with the control experiment. Akt, a serinethreonine kinase that is directly activated in response to PI3Ks, is a major effector of PI3Ks in cancer (9). In this study, the phosphorylation level of Akt at Ser473 (one of the two target amino acids whose phosphorylation upregulates Akt kinase activity) was significantly downregulated in response to capsaicin treatment. Furthermore, downregulated expression of
phospho-PI3 Kinase p85 (Tyr458) and phospho-Akt (Ser473) were observed in capsaicin-treated mice. These results suggest that downregulation of the PI3K/Akt signaling pathway may be involved in capsaicin-induced apoptosis in PANC-1 cells.

Activated Akt phosphorylates several cellular proteins, including glycogen synthase kinase-3 $\alpha$ (GSK-3 $\alpha$ ), GSK-3 $\beta$, forkhead box $\mathrm{O}$ transcription factors (FoxO), murine double minute 2 (MDM2), B-cell lymphoma-2 (BCL2)-interacting mediator of cell death (BIM) and BCL2-associated agonist of cell death (BAD), to facilitate cell survival and cell cycle entry $(8,9)$. However, it remains unknown how activation of the PI3K/Akt signaling pathway promotes cell survival and suppresses apoptosis. It has been demonstrated that inhibition of GSK3 $\beta$, one of the effectors downstream of Akt, leads to G0/G1 phase arrest $(32,33)$. In this study, capsaicin treatment induced G0/G1 phase arrest and downregulation of the PI3K/ Akt pathway in PANC-1 cells. Therefore, downregulation of the PI3K/Akt/GSK3 $\beta$ pathway may be involved in capsaicininduced G0/G1 phase arrest and apoptosis, which indeed needs further investigation.

Together, our studies suggest that capsaicin-induced apoptosis may correlate with downregulation of the PI3K/Akt pathway. Thus, the present study provides novel insights into the molecular mechanisms of capsaicin in panreatic cancer cells. These findings strengthen the idea that capsaicin may be used as an anti-neoplastic medicine and that the PI3K/Akt pathway is a promising target for therapeutic intervention in pancreatic cancer.

\section{Acknowledgements}

The authors are grateful for funding support from the Administration of Traditional Chinese Medicine of Zhengjing Province, China (Grant No. 2011ZZ010), Zhejiang Provincial Science Fund for Distinguished Young Scholars (Grant No. LR12H280001) and The National Natural Science Foundation of China (Grant No. 81173606).

\section{References}

1. Vincent A, Herman J, Schulick R, Hruban RH and Goggins M: Pancreatic cancer. Lancet 378: 607-620, 2011.

2. Raimondi S, Maisonneuve P and Lowenfels AB: Epidemiology of pancreatic cancer: an overview. Nat Rev Gastroenterol Hepatol 6: 699-708, 2009.

3. Wang L, Yang GH, Lu XH, Huang ZJ and Li H: Pancreatic cancer mortality in China (1991-2000). World J Gastroenterol 9: 1819-1823, 2003

4. Li D, Xie K, Wolff R and Abbruzzese JL: Pancreatic cancer. Lancet 363: 1049-1057, 2004.

5. Saif MW: Pancreatic neoplasm in 2011: an update. JOP 12: 316-321, 2011.

6. Stathis A and Moore MJ: Advanced pancreatic carcinoma: current treatment and future challenges. Nat Rev Clin Oncol 7: 163-172, 2010.

7. Arends JJ, Sleeboom HP, Leys MB, et al: A phase II study of raltitrexed and gemcitabine in patients with advanced pancreatic carcinoma. Br J Cancer 92: 445-448, 2005.

8. Liu P, Cheng H, Roberts TM and Zhao JJ: Targeting the phosphoinositide 3-kinase pathway in cancer. Nat Rev Drug Discov 8: 627-644, 2009

9. Engelman JA: Targeting PI3K signalling in cancer: opportunities, challenges and limitations. Nat Rev Cancer 9: 550-562, 2009

10. Datta SR, Dudek H, Tao X, Masters S, Fu H, Gotoh Y and Greenberg ME: Akt phosphorylation of BAD couples survival signals to the cell-intrinsic death machinery. Cell 91: 231-241, 1997. 
11. Fresno Vara JA, Casado E, de Castro J, Cejas P, Belda-Iniesta C and Gonzalez-Baron M: PI3K/Akt signalling pathway and cancer. Cancer Treat Rev 30: 193-204, 2004.

12. Bondar VM, Sweeney-Gotsch B, Andreeff M, Mills GB and McConkey DJ: Inhibition of the phosphatidylinositol 3'-kinase-AKT pathway induces apoptosis in pancreatic carcinoma cells in vitro and in vivo. Mol Cancer Ther 1: 989-997, 2002.

13. Roy SK, Srivastava RK and Shankar S: Inhibition of PI3K/ AKT and MAPK/ERK pathways causes activation of FOXO transcription factor, leading to cell cycle arrest and apoptosis in pancreatic cancer. J Mol Signal 5: 10, 2010.

14. Surh YJ: More than spice: capsaicin in hot chili peppers makes tumor cells commit suicide. J Natl Cancer Inst 94: 1263-1265, 2002.

15. Surh YJ,Lee E and Lee JM: Chemoprotective properties of some pungent ingredients present in red pepper and ginger. Mutat Res 402: 259-267, 1998.

16. Caterina MJ, Schumacher MA, Tominaga M, Rosen TA, Levine JD and Julius D: The capsaicin receptor: a heat-activated ion channel in the pain pathway. Nature 389: 816-824, 1997.

17. Hartel M, di Mola FF, Selvaggi F, et al: Vanilloids in pancreatic cancer: potential for chemotherapy and pain management. Gut 55: 519-528, 2006.

18. Pramanik KC, Boreddy SR and Srivastava SK: Role of mitochondrial electron transport chain complexes in capsaicin mediated oxidative stress leading to apoptosis in pancreatic cancer cells. PLoS One 6: e20151,2011.

19. Amantini C, Ballarini P, Caprodossi S, et al: Triggering of transient receptor potential vanilloid type 1 (TRPV1) by capsaicin induces Fas/CD95-mediated apoptosis of urothelial cancer cells in an ATM-dependent manner. Carcinogenesis 30 1320-1329, 2009.

20. Kim JY, Kim EH, Kim SU, Kwon TK and Choi KS: Capsaicin sensitizes malignant glioma cells to TRAIL-mediated apoptosis via DR5 upregulation and survivin downregulation. Carcinogenesis 31: 367-375, 2010.

21. Ito K, Nakazato T, Yamato K, et al: Induction of apoptosis in leukemic cells by homovanillic acid derivative, capsaicin, through oxidative stress: implication of phosphorylation of $\mathrm{p} 53$ at Ser-15 residue by reactive oxygen species. Cancer Res 64: 1071-1078, 2004.
22. Sanchez AM, Sanchez MG, Malagarie-Cazenave S, Olea N and Diaz-Laviada I: Induction of apoptosis in prostate tumor PC-3 cells and inhibition of xenograft prostate tumor growth by the vanilloid capsaicin. Apoptosis 11: 89-99, 2006.

23. Zhang R, Humphreys I, Sahu RP, Shi Y and Srivastava SK: In vitro and in vivo induction of apoptosis by capsaicin in pancreatic cancer cells is mediated through ROS generation and mitochondrial death pathway. Apoptosis 13: 1465-1478, 2008.

24. Shin DH, Kim OH, Jun HS and Kang MK: Inhibitory effect of capsaicin on B16-F10 melanoma cell migration via the phosphatidylinositol 3-kinase/Akt/Racl signal pathway. Exp Mol Med 40: 486-494, 2008

25. Wei WT, Chen H, Wang ZH, et al: Enhanced antitumor efficacy of gemcitabine by evodiamine on pancreatic cancer via regulating PI3K/Akt pathway. Int J Biol Sci 8: 1-14, 2012.

26. Wei WT, Chen H, Ni ZL, et al: Antitumor and apoptosispromoting properties of emodin, an anthraquinone derivative from Rheum officinale Baill, against pancreatic cancer in mice via inhibition of Akt activation. Int J Oncol 39: 1381-1390, 2011.

27. Shanmugam MK, Kannaiyan R and Sethi G: Targeting cell signaling and apoptotic pathways by dietary agents: role in the prevention and treatment of cancer. Nutr Cancer 63: 161-173, 2011.

28. Sarkar FH and Li Y: Using chemopreventive agents to enhance the efficacy of cancer therapy. Cancer Res 66: 3347-3350, 2006.

29. Degterev A, Boyce M and Yuan J: A decade of caspases. Oncogene 22: 8543-8567, 2003.

30. Hamacher R, Schmid RM, Saur D and Schneider G: Apoptotic pathways in pancreatic ductal adenocarcinoma. Mol Cancer 7: 64, 2008.

31. Kasibhatla $\mathrm{S}$ and Tseng B: Why target apoptosis in cancer treatment? Mol Cancer Ther 2: 573-580, 2003.

32. Hashimoto T, He Z, Ma WY, Schmid PC, Bode AM, Yang CS and Dong Z: Caffeine inhibits cell proliferation by G0/G1 phase arrest in JB6 cells. Cancer Res 64: 3344-3349, 2004.

33. Liang J and Slingerland JM: Multiple roles of the PI3K/PKB (Akt) pathway in cell cycle progression. Cell Cycle 2: 339-345, 2003. 